\title{
The boundedness principle characterizes second category subsets
}

\section{Kevin A. Broughan}

\begin{abstract}
Converses are proved for the Osgood (the Principle of Uniform Boundedness), Dini, and other well known theorems. The notion of a continuous step function on a topological space is defined and a class of spaces identified for which each lower semicontinuous function is the pointwise limit of a monotonically increasing sequence of step functions.
\end{abstract}

\section{Introduction}

Let $X$ and $Y$ be $T_{1}$ topological spaces. Thornton has proved in [4] that $X$ is homeomorphic to $Y$ if and only if the semiring of positive lower semicontinuous functions on $X$ is isomorphic to the semiring of positive lower semicontinuous functions on $Y$. (His theorem is stronger than this.) Because of this result many topological properties will have equivalent formulations in terms of lower semicontinuous functions. We illustrate this idea by characterizing countably compact topological spaces and subsets of the second category in topological spaces. We include a theorem showing that a positive lower semicontinuous function on a Čech dimension zero metrizable space can be expressed as the pointwise limit of a sequence of monotonically increasing locally constant functions.

Recejved 25 November $17 \% 6$. 


\section{Boundedness principle}

The statement of a well known boundedness principle is as follows. Let $(X, \tau)$ be a topological space and let $S$ be a nonempty second category subset of $X$. Let $\left(f_{\lambda}\right)_{\lambda \in \Lambda}$ be a family of lower semicontinuous functions on $X$ with the following property: for each $x$ in $S$ there is a real number $m_{x}$ with $f_{\lambda}(x) \leq m_{x}$ for all $\lambda$ in $\Lambda$. Then there exists a nonempty open subset $P$ of $X$ and a real number $M$ such that $f_{\lambda}(x) \leq M$ for all $\lambda$ in $\Lambda$ and $x$ in $P$. Theorem 1 provides a converse to this theorem.

THEOREM 1. Let $(X, \tau)$ be a topological space and let $S \subset X$ be a nonempty subset. Then the following conditions are equivalent:

(I) the subset $S$ is of the second category in $X$; and

(2) for each family $\left(f_{\lambda}\right)_{\lambda \in \Lambda}$ of lower semicontinuous functions which is pointwise bounded on $S$ there is a nonempty open subset $P$ of $X$ and a number $M$ such that $f_{\lambda}(x) \leq M$ for alz $\lambda$ in $\Lambda$ and $x$ in $P$.

Proof. (1) $\Rightarrow(2)$. This is known as the Uniform Boundedness Principle or Osgood's Theorem [3, Theorem 17, p. 140].

$(2) \Rightarrow(1)$. Let $S=\bigcup_{i=1}^{\infty} S_{i}$ where each $\bar{S}_{i}$ has an empty interior. For each $n$ in $N$, the positive integers, let $A_{n}=X \sim \bigcup_{i=1}^{n} \bar{S}_{i}$ and let

$$
f_{n}(x)=n \chi_{A_{n}}(x)
$$

that is, $f_{n}$ is $n$ times the characteristic function of the complement of $\bigcup_{i=1}^{n} \bar{S}_{i}$. Then, for each $n, f_{n}$ is lower semicontinuous on $X$. Let
$x \in S$ and let $j_{x}$ be the first integer $j$ with the property $x \in \bar{S}_{j}$.
If $m_{x}=j_{x}-1$, then $f_{n}(x) \leq m_{x}$ for all $n$ in $\mathrm{N}$. Therefore the
family of functions $\left(f_{n}\right)_{n \in \mathrm{N}}$ is pointwise bounded on $S$. By hypothesis 
there is a real number $M$ and a nonempty open set $P$ with $f_{n}(x) \leq M$ for all $n$ and all $x$ in $P$. If $t \in N$ is greater than $M$ then $f_{t}(x)<t$ for all $x$ in $P$. This implies $P \subset \bigcup_{i=1}^{t} \bar{S}_{i}$, a contradiction. Therefore $S$ is of the second category in $X$. This completes the proof.

THEOREM 2. Let $(X, \tau)$ be a perfectly normal topological space and let $S \subset X$ be a nonempty subset. Then the following conditions are equivalent:

(1) the subset $S$ is of the second category in $X$; and

(2) each family of continuous functions on $X$ which is pointwise bounded from above on $S$ is uniformly bounded on some nonempty open subset of $X$.

Proof. (1) $\Rightarrow(2)$. This follows immediately from Theorem 1 above.

(2) $\Rightarrow(1)$. Suppose that $S$ has the given property but is not of the second category in $X$. Then there exists a family of nonempty sets $\left(S_{n}\right)_{n \in \mathrm{N}}$ with $\bar{S}_{n}^{0}=\emptyset$ for each $n, S_{n} \subset S_{n+1}$ for each $n$ and $\bigcup_{n=1}^{\infty} S_{n}=S$. Because each $\bar{S}_{n}$ is a $G_{\delta}$ there exists a denumerable family $\left(G_{n, j}\right)_{j \in N}$ of open subsets of $X$ satisfying $\bar{S}_{n}=\bigcap_{j=1}^{\infty} G_{n, j}$ and $G_{n, j} \supset G_{n, j+1}$ for each $j$ in $N$. For each $n$ and $j$ there is a continuous function $\varphi_{n, j}: X \rightarrow[0,1]$ satisfying $\varphi_{n, j}\left(\bar{S}_{n}\right)=0$ and $\varphi_{n, j}\left(X-G_{n, j}\right)=1$.

Define a family of continuous functions on $X$ by the rule $f_{n, j}(x)=n \varphi_{n, j}(x)$ for each $x$ in $X$.

If $x \in S$ let $n_{x}$ be the first integer satisfying $x \in S_{n_{x}}$. Then $\varphi_{n, j}(x)=0$ for all $n \geq n_{x}$ and therefore $f_{n, j}(x) \leq n_{x}-1$ for all $n$ and $j$. Thus $\left(f_{n, j}\right)$ is pointwise bounded on $S$. Therefore there exists a number $M$ and a nonempty open subset $P \subset X$ such that 


$$
f_{n, j}(x) \leq M \text { for all } x \text { in } P \text { and } n, j \text { in } \mathrm{N} \text {. }
$$

In particular if $n>M$ and $x \in P$ then $\varphi_{n, j}(x)<1$ for all $j$ in $N$. Thus, if $n>M, P \subset \prod_{j=1}^{\infty} G_{n, j}=\bar{S}_{n}$, which is a contradiction. Therefore $S$ is of the second category in $X$. Let $X$ be a perfectly normal space and let $E \subset C(X)$ be a subspace of the space of continuous real valued functions on $X$. We say $E$ is a test space for second category subsets of $X$ if the following condition is satisfied: whenever $S \subset X$ is such that any family $F \subset E$ which is pointwise bounded on $S$ is uniformly bounded on a nonempty open subset of $X$, then $S$ is necessarily of the second category in $X$. For specific spaces $X$ it is possible to find test spaces which are smaller than $C(X)$ itself.

THEOREM 3. The space of analytic functions on $\mathrm{R}^{n}$ is a test space for second category subsets of $R^{n}$.

Proof. (a) The space $C^{\infty}\left(\mathrm{R}^{n}\right)$ is a test space for $\mathrm{R}^{n}$ : let $S \subset \mathrm{R}^{n}$ and suppose that subfamilies of $C^{\infty}(\mathrm{R})$ which are pointwise bounded on $S$ are uniformly bounded on open subsets of $R^{n}$. Let $F \subset C\left(R^{n}\right)$. For each $f$ in $F$ there is a $g_{f}$ in $C^{\infty}\left(R^{n}\right)$ with $|f(x)-g(x)| \leq 1$ for all $x$ in $\mathrm{R}^{n}$. Let $G=\left\{g_{f}: f \in F\right\}$. Then because $G$ is uniformly bounded on an open subset of $\mathrm{R}^{n}$, so also is $F$. Thus $S$ is a second category subset. This proves (a).

(b) The space $D\left(\mathrm{R}^{n}\right)$ of functions in $C^{\infty}\left(\mathrm{R}^{n}\right)$ with compact support is a test space for $\mathrm{R}^{n}$ : let $F \subset C^{\infty}\left(\mathrm{R}^{n}\right)$ be pointwise bounded on $S$. For each $m$ in $N$ let $\lambda_{m} \in D\left(R^{n}\right)$ satisfy $0 \leq \lambda_{m}(x) \leq 1$ for all $x$, $\lambda_{m}(x)=0$ when $\left|x^{\prime}\right| \geq m+1$ and $\lambda_{m}(x)=1$ when $|x| \leq m$. Let $G=\left\{\lambda_{m} \cdot f: m \in N, f \in F\right\}$. Then $G \subset D\left(R^{n}\right)$ is uniformly bounded on an open subset of $\mathrm{R}^{n}$ and therefore $F$ is also. Thus, by (a), $S$ is second category. Therefore $D\left(\mathrm{R}^{n}\right)$ is a test space for second category subset of 
$\mathrm{R}^{n}$

(c) The space $C^{\omega}\left(R^{n}\right)$ of analytic functions on $R^{n}$ is a test space. Let $\psi(x)=\exp \left(-|x|^{2}\right)$ when $x \in R^{n}$. We will prove that the algebra $A$ generated by $\{1, \psi\}$ is a test space of $R^{n}$. This will prove (c) as if $E$ and $F$ are subspaces of $C(X)$ with $E \subset F$ and $E$ is a test space then $F$ is also a test space. Let $S \subset \mathrm{R}^{n}$ and let $F \subset D\left(\mathrm{R}^{n}\right)$. By an extension of the Stone-Weierstrass Theorem, for each $f$ in $F$ there is a $g_{f}$ in $A$ with $\left|f(x)-g_{f}(x)\right| \leq 1$ for all $x$ in $\mathrm{R}^{n}$. The proof of (c) follows from this. This completes the proof.

COMPACTNESS PRINCIPLE. We will prove the converse to the following theorem. If $f$ is a lower semicontinuous function on a countably compact space $(X, \tau)$ then $f$ is bounded from below.

THEOREM 4. Let $(X, \tau)$ be a topological space. Then the following conditions are equivalent:

(1) the space $X$ is countably compact; and

(2) every Lower semicontinuous function on $X$ is bounded from below.

Proof. (I) $\Rightarrow(2)$. See [3, Proposition 10, p. 161].

$(2) \Rightarrow(1)$. Suppose that $X$ is not countably compact but satisfies the hypothesis of the theorem. Then there exists a countable family $\left(o_{n}\right)_{n \in \mathbb{N}}$ of nonempty open subsets of $X$ with $o_{n} \subset o_{n+1}$ for all $n$, $X=\bigcup_{n=1}^{\infty} o_{n}$ and such that $X$ is not equal to $\bigcup_{n=1}^{i} o_{n}$ for any integer $i$. Define a family of lower semicontinuous functions as follows:

$$
f_{n}(x)=\sum_{i=1}^{n} x_{0}(x)-n
$$

where $x_{O_{i}}$ is the characteristic function of $o_{i}$ for each $i$ in $N$. Then $f_{n+1}(x) \leq f_{n}(x)$ for all $x$ and all $n$.

Let $x \in X$ and let $j_{x}$ be the first $j$ in $N$ for which $x \in o_{j}$. 
Then if $1 \leq n<j_{x}$,

$$
f_{n}(x)=-n \geq 1-j_{x}
$$

If $j_{x} \leq n$ then

$$
f_{n}(x)=\left\{\sum_{i=j}^{i=n} 1\right\}-n=1-j_{x} .
$$

Thus $\lim _{n \rightarrow \infty} f_{n}(x)=1-j_{x}$ for each $x$ in $x$. Let $f(x)=1-j_{x}$. We will prove that $f$ is lower semicontinuous on $X$. Let $x \in X$ and let $\varepsilon>0$ be given. If $y \in o_{j_{x}}$ then $j_{y} \leq j_{x}$ and thus, for sufficiently large $n, f_{n}(y)=1-j_{y} \geq 1-j_{x}=f_{n}(x)$. Therefore $f(y)>f(x)-\varepsilon$ for each $y$ in $o_{j_{x}}$ and thus $f$ is lower semicontinuous on $X$. But $f$ is not bounded from below. This contradiction completes the proof.

THEOREM 5. Let $(X, \tau)$ be a normal topological space. Then the following conditions are equivalent:

(1) $X$ is countably compact; and

(2) each continuous function on $X$ is bounded from below.

Proof. (1) $\Rightarrow(2)$. See Theorem 4.

(2) $\Rightarrow$ (1). Any pseudo compact normal space is countably compact.

THEOREM 6. Let $(X, \tau)$ be a topological space. Then the following conditions are equivalent:

(1) $X$ is countably compact; and

(2) if $\left(f_{n}\right)$ is a sequence of upper semicontinuous functions on $X$ which converges monotonically to zero at each point of $x$ then $\left(f_{n}\right)$ converges uniformly to zero on $X$.

Proof. (1) $\Rightarrow(2)$. See Dini's Theorem [3, Proposition 11, p. 162].

$(2) \Rightarrow(1)$. Let $\left(F_{n}\right)$ be a nested sequence of closed sets in $X$ with 
$\infty$

$\bigcap_{n=1} F_{n}=\emptyset$. Let $f_{n}=\chi_{F_{n}}$, the characteristic function of $F_{n}$ for each $n$ in $N$. Then $f_{n}(x) \rightarrow 0$ monotonically for each $x$ in $X$. By (2) there exists an $N$ in $N$ such that $f_{n}(x)<\frac{1}{2}$ for all $x$ and $n \geq N$. But this means $\bigcap_{n=1}^{N} F_{n}=\varnothing$, and therefore $X$ is countabIy compact.

THEOREM 7. Let $X$ be a perfectly normal topological space. Then the following conditions are equivalent:

(1) $X$ is countably compact; and

(2) if $\left(f_{n}\right)$ is a sequence of continuous functions on $X$ which converge montonically to zero at each point of $X$ then $\left(f_{n}\right)$ converges uniformly to zero on $X$.

Proof. We need only show that $(2) \Rightarrow(1)$. Let $\left(F_{n}\right)$ be a nested sequence of closed sets in $X$ with $\bigcap_{n=1}^{\infty} E_{n}=\varnothing$. For each $n$ let $\left(G_{n, j}\right)_{j \in N}$ be a nested family of open subsets with $F_{n}=\bigcap_{j=1}^{\infty} G_{n, j}$. Let $\varphi_{n, j}: X \rightarrow[0,1]$ be a continuous function satisfying $\varphi_{n, j}\left(F_{n}\right)=1$ and $\varphi_{n, j}\left(X-G_{n, j}\right)=0$ for each $n$ and $j$ in $\mathrm{N}$. Let

$$
f_{n}=\min \left\{\varphi_{n, j}: 1 \leq i, j \leq n\right\}
$$

Then $\left(f_{n}(x)\right)$ converges monotonically to zero for each $x$ in $X$. By (2) there exists an integer $N$ such that $f_{n}(x)<\frac{7}{2}$ for each $x$ in $X$ and $n \geq N$. But then necessarily $\prod_{n=1}^{N} F_{n}=\varnothing$. This completes the proof that $X$ is countably compact.

\section{Step functions}

Let $X=[a, b]$ be a closed interval of real numbers with the usual topology. A step function on $X$ can be made into a lower semicontinuous 
step function by altering its values if necessary at the endpoints of the subintervals of $X$ upon which it takes constant values. The following theorem characterizes lower semicontinuous functions on $X$. Let $f$ be a real valued function on $X$. Then $f$ is lower semicontinuous if and only if it is the pointwise limit of a monotonically increasing sequence of lower semicontinuous step functions. We will define the notion step function for a different class of spaces and prove an analogous theorem.

Let $(X, \tau)$ be a metrizable space having large inductive dimension zero. Then there exists a sieve for $X,[1]$; that is to say a family $\left(D_{n}\right)$ of open covers of $X$, where $D_{n}=\left(B_{n, \lambda}\right)_{\lambda \in I_{n}}$, satisfying

(i) $D_{n+1}$ refines $D_{n}$ for all $n$,

(ii) $B_{n, \lambda} \cap B_{n, \gamma}=\varnothing$ if $\lambda \neq \gamma$, and

(iii) $\left(D_{n}\right)$ is a base for the topology $\tau$.

We say a function $\varphi: X \rightarrow R$ is a step function if it is locally constant; that is, $\varphi^{-1} \varphi(x)$ is open for each $x$ in $X$. Then each step function is continuous. The spaces for which these functions are interesting are clearly highly disconnected. We do not place restrictions on the range of $\varphi$. If $\left\{\varphi_{n}\right\}$ is a monotonically increasing sequence of step functions and $\lim _{n \rightarrow \infty} \varphi_{n}(x)=f(x)<\infty$ for each $x$ in $X$ then $f(x)$ is a real valued lower semicontinuous function on $X$. We will show that every positive lower semicontinuous function arises in this manner.

THEOREM 8. Let $(X, \tau)$ be a metrizable space. Then the following conditions are equivalent:

(1) X has large inductive dimension zero; and

(2) if $f$ is a positive, real valued, lower semicontinuous function on $X$ then there exists a monotonically increasing sequence $\left\{\varphi_{n}\right\}$ of positive step functions on $X$ with $f(x)=\lim _{n \rightarrow \infty} \varphi_{n}(x)$ for each $x$ in $X$.

Proof. (1) $\Rightarrow(2)$. Let $D=\left(B_{n, \lambda}: n \in N, \lambda \in I_{n}\right)$ be a compatible 
sieve for $X$. If $n \in N$ and $x \in B_{n, \lambda}$ let

$$
\varphi_{n}(x)=\inf \left\{f(y): y \in B_{n, \lambda}\right\} \text {. }
$$

Then $\varphi_{n}$ is a step function and $\varphi_{n}(x) \leq \varphi_{n+1}(x) \leq f(x)$. We will prove that $\lim _{n \rightarrow \infty} \varphi_{n}(x)=f(x)$. Given $\varepsilon>0$ and $x$ in $X$ there is an open set 0 with $x$ in 0 and $f(y)>f(x)-\varepsilon$ for $y$ in 0 . There is an $n$ in $N$ and a $\lambda \in I_{n}$ with $x \in B_{n, \lambda} \subset 0$. Then

$$
\varphi_{n}(x)=\inf \left\{f(y): y \in B_{n, \lambda}\right\} \geq f(x)-\varepsilon .
$$

Thus $\lim _{n \rightarrow \infty} \varphi_{n}(x)=f(x)$ for each $x$ in $X$. This completes the proof.

$(2) \Rightarrow(1)$. Let $P \simeq X$ be a nonempty open set and let $f$ be the characteristic function of $P$. If $\left\{\varphi_{n}\right\}$ is the sequence of step functions given by (2) let $B_{n}=\left\{x \in X: \varphi_{n}(x) \geq 1-(1 / n)\right\}$. Then $B_{n}$ is open and closed and $P=\bigcup_{n=1}^{\infty} B_{n}$. Since this is true for any open subset we can transform a $\sigma$-locally finite base for $X$ into a $\sigma$-locally finite base consisting of sets which are both open and closed. Thus, by [2, Theorem 5, p. 291], $X$ has large inductive dimension zero.

\section{References}

[1] K.A. Broughan, "Metrization of spaces having Čech dimension zero", Buzl. Austral. Math. Soc. 9 (1973), 161-168.

[2] R. Engelking, Outline of general topology (translated by K. Sieklucki. North-Holland, Amsterdam; PWN-Polish Scientific Publishers, Warsaw; 1968).

[3] H.L. Royden, Real analysis, second edition (Macmillan, New York; Collier-Macmillan, London; 1968).

[4] Melvin C. Thornton, "Characterizing topologies by functions", Proc. Amer. Wath. Soc. 36 (1972), 613-614.

Department of Mathematics, University of Waikato, Hamilton, New Zealand. 